\title{
In vivo evaluation of an antibacterial coating containing halogenated furanone compound- loaded poly(L-lactic acid) nanoparticles on microarc-oxidized titanium implants
}

\author{
This article was published in the following Dove Press journal: \\ International Journal of Nanomedicine \\ 30 March 2016 \\ Number of times this article has been viewed
}

\author{
Yicheng Cheng ${ }^{1-3, *}$ \\ Bo $\mathrm{Gao}^{1,3, *}$ \\ Xianghui Liu',* \\ Xianghui Zhao ${ }^{4}$ \\ Weige Sun ${ }^{2}$ \\ Huifang Ren ${ }^{1,3}$ \\ Jiang $\mathrm{Wu}^{1,3}$
}

'Department of Prosthodontics, State Key Laboratory of Military Stomatology, School of Stomatology, Fourth Military Medical University, Xi'an, ${ }^{2}$ Department of Stomatology, Bayi Hospital, Nanjing University of Chinese Medicine, Nanjing, ${ }^{3}$ Department of Prosthodontics, Shaanxi Key Laboratory of Stomatology, Xi'an, ${ }^{4}$ Institute of Neuroscience, School of Basical Medical Science, Fourth Military Medical University, Xi'an, People's Republic of China

*These authors contributed equally to this work

Correspondence: jiang Wu Department of Prosthodontics, State Key Laboratory of Military Stomatology, School of Stomatology, Fourth Military Medical University, No I45, West Changle Road, Xi'an, Shaanxi 710032, People's Republic of China

Tel/fax +86298477 6469

Email wujiang@fmmu.edu.cn

\begin{abstract}
To prevent peri-implant infection, a new antibacterial coating containing a halogenated furanone compound, ( $Z$-)-4-bromo-5-(bromomethylene)-2(5H)-furanone-loaded poly(L-lactic acid) nanoparticles, has been fabricated. The current study was designed to evaluate the preventive effect of the antibacterial coating under a simulated environment of peri-implant infection in vivo. Microarc-oxidized titanium implants treated with minocycline hydrochloride ointment were used as positive control group, and microarc-oxidized titanium implants without any treatment were used as blank control group. Three kinds of implants were implanted in dogs' mandibles, and the peri-implant infection was simulated by silk ligation and feeding high sugar diet. After 2-month implantation, the results showed that no significant differences were detected between the experimental and positive control groups $(P>0.05)$, but the data of clinical measurements of the blank control group were significantly higher than those of the other two groups $(P<0.05)$, and the bone-implant contact rate and ultimate interfacial strength were significantly lower than those of the other two groups $(P<0.05)$. Scanning electron microscope observation and histological examination showed that more new bone was formed on the surface of the experimental and positive control groups. It can be concluded that the antibacterial coating fabricated on implants has remarkable preventive effect on peri-implant infection at the early stage.
\end{abstract}

Keywords: antibacterial coating, halogenated furanone, microarc-oxidized, nanoparticles, in vivo

\section{Introduction}

Dental implantation techniques have been firmly established as an integral part of oral rehabilitation treatment. Despite the high success rates, ${ }^{1,2}$ dental implant failure may occur and is defined as the inadequacy of the tissues to establish or maintain the osseointegration. ${ }^{3,4}$ Bacterial-associated peri-implant infection is considered an inflammatory process affecting the tissues around the implant in function, resulting in loss of supporting bone, and is reported to be one of most important reasons causing dental implant failure. ${ }^{5}$ The oral cavity is a complex microecological environment, and ${ }^{6}$ after implantation, the bacteria can invade the peri-implant soft tissue and may potentially progress deeper into the bone and undermine the osseointegration process. ${ }^{7}$ Once infections occur, bacteria tend to aggregate in a hydrated polymeric matrix to form biofilm on the implant surface, which protects bacteria from the host defense system and antibacterial agents coming from outside via several mechanisms. ${ }^{89}$ A simple and promising approach to reduce the risk of peri-implant infection is fabricating 
antimicrobial surfaces through specific treatments to prevent initial bacterial adhesion. ${ }^{10}$ In previous studies, various antibacterial coatings containing many kinds of antibacterial agents, including antibiotics, proteins, silver, and specific polymers, have been designed onto implant surfaces. ${ }^{11-13}$

Before antibacterial agent-loaded coating on titanium (Ti) implants can be applied clinically, there are still many outstanding issues that need to be solved. First, the susceptibility of bacteria to the antibacterial agent in periimplant is a problem. Drug resistance of bacteria isolated from subgingival species after antibiotic therapy has been reported. ${ }^{14}$ Second, most antibacterial agent-loaded coatings failed to sustain long-term antibacterial effects. ${ }^{10}$ Longterm antibacterial ability is especially significant for dental implants because of the constant risk of bacterial infection. Third, the antibacterial agents should be highly biocompatible. There are references documenting that some types of antibacterial agents may harm cell functions. For instance, ciprofloxacin at a concentration $>100 \mathrm{mg} / \mathrm{mL}$ and vancomycin and tobramycin at a concentration $>2,000 \mathrm{mg} / \mathrm{mL}$ severely decreased cellular proliferation, ${ }^{15}$ and clindamycin at a concentration $>500 \mathrm{mg} / \mathrm{mL}$ had cytotoxic effects on human osteoblasts. ${ }^{16}$ Thus, choosing effective and biocompatible antibacterial agents to incorporate into the coating and rendering the coating to sustain long-term antibacterial effects are urgently needed.

It has been demonstrated that halogenated furanones, originally extracted from the red alga Delisea pulchra, have potent antimicrobial activity against various bacterial species. ${ }^{17,18}$ Halogenated furanones are structurally similar to bacterial $N$-acyl homoserine lactone, which is a signal molecule used for bacterial cell-to-cell communication to control population density and biofilm formation. ${ }^{19}$ Therefore, halogenated furanones acting as $N$-acyl homoserine lactone antagonists inhibit microbial colonization, and the antimicrobial mechanism would render halogenated furanones less prone to the development of drug resistance. ${ }^{20}$ Moreover, studies have indicated that halogenated furanones possess excellent biocompatibility. ${ }^{21}$ Based on these advantages, we have developed a new antibacterial coating containing a halogenated furanone compound, (Z-)-4Bromo-5-(bromomethylene)-2(5H)-furanone (BBF)-loaded poly(L-lactic acid) (PLLA) nanoparticles (BBF-PLLA-NPs) on microarc-oxidized (MAO) Ti in our previous study. ${ }^{22}$ The results confirmed that the antibacterial coating could sustain the release of BBF and showed excellent antibacterial effects for 60 days in vitro. ${ }^{23}$ In this scenario, the current study was specifically designed to further evaluate the preventive effect of the antibacterial coating under a simulated environment of peri-implant infection in vivo.

\section{Materials and methods Fabrication of antibacterial coating containing BBF-PLLA-NPs on MAOTi implants}

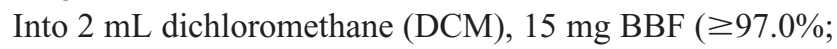
Sigma-Aldrich Co., St Louis, MO, USA) and $100 \mathrm{mg}$ PLLA (number average 99,000 and average molecular weight 152,000 Da; Sigma-Aldrich Co.) were codissolved to form the oil phase, while $40 \mathrm{~mL}$ of $1 \%(\mathrm{w} / \mathrm{v})$ poly(vinyl alcohol) (molecular weight [MW] 89,000-98,000; Sigma-Aldrich Co.) aqueous solution was used as the water phase. Oil phase was added dropwise into the water phase, and the mixture solution was probe sonicated in an ice bath by Sonifier Cell Disrupter (intensity: $300 \mathrm{~W}$ and time: 200 seconds, JY98-IIIN; Ningbo Xingzhi Biological Technology Co., Ltd., Ningbo, People's Republic of China). The resulting emulsion was stirred using a magnetic stirring device (90-1; KaiPeng Experiment Instrument Co., Ltd., Zhengzhou, People's Republic of China) for 6 hours at $25^{\circ} \mathrm{C}$ under reduced pressure to allow the DCM to evaporate completely. Finally, the NPs were collected by centrifugation, washed thrice with distilled water, isolated by centrifugation, and freeze dried. ${ }^{22}$ Scanning electron microscopy (SEM, S-4800; Hitachi Ltd., Tokyo, Japan) was used to evaluate the surface morphology of the NPs. The samples were dried and sputter coated with platinum prior to scanning electron microscope examination. Besides, the mean particle size and size distribution were assessed by dynamic light scattering using a particle size analyzer (SALD-7101; Shimadzu Corporation, Tokyo, Japan) at $25^{\circ} \mathrm{C}$.

Twenty-four smooth cylindrical commercial pure $\mathrm{Ti}$ implants (3.5 mm diameter and $10 \mathrm{~mm}$ length; Ruige Co., Ltd., Baoji, People's Republic of China) were prepared. In a mixed aqueous solution containing $0.2 \mathrm{M}$ calcium acetate and $0.04 \mathrm{M} \beta$-glycerophosphate sodium, the cylindrical implants were MAO treated by a pulsed direct current power supply (applied voltage: $300 \mathrm{~V}$, frequency: $600 \mathrm{~Hz}$, duty cycle: $8.0 \%$, and oxidizing time: 5 minutes; Xi'an University of Technology, Xi'an, People's Republic of China). After MAO treatment, $20 \mathrm{mg}$ of BBF-PLLA-NPs was sonicated in $5 \mathrm{~mL}$ of $0.2 \%$ gelatin solution $(\mathrm{w} / \mathrm{v})$, from which $400 \mu \mathrm{L}$ suspensions was dropped onto eight randomized selected MAO Ti implants (the BBF actually incorporated in the coating was $\sim 0.148 \mathrm{mg} / \mathrm{cm}^{2}$ of the implant area). The implants were then oscillated on an oscillator (HY-2; Huier Experiment Instrument Co., Ltd., Hangzhou, People's 
Republic of China) for 1 hour to enable the NPs to penetrate the pores present on the surface of the MAO Ti implants. Finally, the implants were dried at $4^{\circ} \mathrm{C}$, immersed in $2.5 \%$ $(\mathrm{w} / \mathrm{v})$ glutaraldehyde solution for 30 minutes, washed with ethanol thrice, and sterilized by $\mathrm{Co}^{60}$ radiation. The detailed processes were recorded in our previous study. ${ }^{22}$ Only MAOtreated Ti implants were set as control. The surface morphology of smooth Ti implant, MAO-treated Ti implants, and the antibacterial coating containing BBF-PLLA-NPs were observed by scanning electron microscopy (SEM).

\section{Surgical procedures}

Six adult male Beagle dogs, each weighing 12-15 kg, were used in this study. The protocol was approved by the Animal Care and Use Committee, School of Stomatology, Fourth Military Medical University. All surgical procedures were performed under systemic ( $1 \mathrm{mg} / \mathrm{kg}$ pentobarbital sodium) and local (2\% lidocaine with 1:80,000 epinephrine) anesthesia. Prior to surgery, the dogs received Gentocin ${ }^{\circledR}\left(2 \mathrm{~cm}^{3}\right)$ subcutaneously as an antibiotic prophylaxis and continued to receive Gentocin for 7 days post-surgery. Six dogs were randomly divided into the following three groups: experimental group (MAO Ti implants coated with the antibacterial coating), positive control group (MAO Ti implants treated with minocycline hydrochloride ointment, once a week after implantation), and blank control group (MAO Ti implants). The surgical procedures included tooth extraction surgery and implantation surgery. After routine disinfection, both mandible third premolars of each dog were extracted using standard oral surgery techniques with elevators, forceps, and a high-speed handpiece. Then a cylindrical implant system (Friatec AG, Mannheim, Germany) was used to implant immediately. Four implants of each group were inserted into one dog (two implants in unilateral teeth extraction sockets). The implants were inserted in appropriate depth to ensure the implant exposure $\sim 2 \mathrm{~mm}$ upon the gums. The surgical area was liberally irrigated with normal saline to remove bone fragments, and the tissue flaps were closed with continuous sutures. The peri-implant infection was simulated by ligaturing 4-0 silk around the implants upon the gums and feeding high sugar diet $(100 \mathrm{~g} / \mathrm{kg})$. All the operations are performed by a skilled dentist.

\section{Clinical measurements}

After 2 months of implantation, clinical measurements were performed. Probing depth (PD) was measured by a periodontal probe at four points (buccal, lingual, mesial, and distal sides) around the implants to measure the depth of peri-implant pocket. The peri-implant sulcular fluid (PISF) volume was measured by a Periotron (Harco Electronics, Dental Products Division, Winnipeg, Canada). Periopaper (Harco Electronics) was inserted in the implant sulcus for 30 seconds, and then it was placed between the upper and lower counterparts of the Periotron. The volume of PISF was measured as Periotron units. And the mobility of implants (MOI) was measured by Periotest (Siemens, Bensheim, Germany). All the data were recorded and calculated.

The X-ray radiographs of the mandible third premolars before extraction, after implantation immediately, and after 2-month implantation were carried out.

\section{Bone-implant interface observation}

After 2 months of implantation, all the dogs were euthanized, and the mandibles were removed. The implants were recovered by sectioning the bone $\sim 10 \mathrm{~mm}$ medial and distal to the implant center. For observation of the interface between bone and implant, different groups of implants were immediately fixed in 4\% formaldehyde solution for 3 days after sacrifice. The specimens were dehydrated in graded ethanol solution (75\%, 95\%, and 100\%, increasing every 3 days) and embedded in poly(methyl methacrylate) resin. Then they were cut along the long axis of the implants using a diamond blade, and the section closest to the longitudinal mid-sagittal plane was selected, ground down to a thickness of $100 \mathrm{~mm}$, and polished. Some of the histological sections were observed under SEM. The others were stained with Stevenel's blue and Van Gieson's picrofuchsin. Histomorphometry was carried out using an image processing system (Leica DM 6000B; Leica Microsystems, Wetzlar, Germany). The boneimplant contact (BIC) rate was measured by image analysis techniques (CS-100A; Leica Microsystems). The BIC levels were defined as the fraction of direct bone apposition at the surface of the implant.

\section{Pull-out test}

Pull-out test was performed using an electronic universal material test machine (AG-X; Shimadzu Corporation) to evaluate the ultimate interfacial strength of the implants at the bone-implant interface. The procedures of the pull-out test are shown in Figure 1. In brief, the implant-bone blocks were embedded in poly(methyl methacrylate) resin and ensure that the implants did not contact the poly(methyl methacrylate) resin directly (Figure 1A). Then the sample was fixed in a tensile test box (Figure 1B). Finally, the box was stabilized in the electronic universal material test machine (Figure 1C), and the implants were pulled out along the axial at a speed 

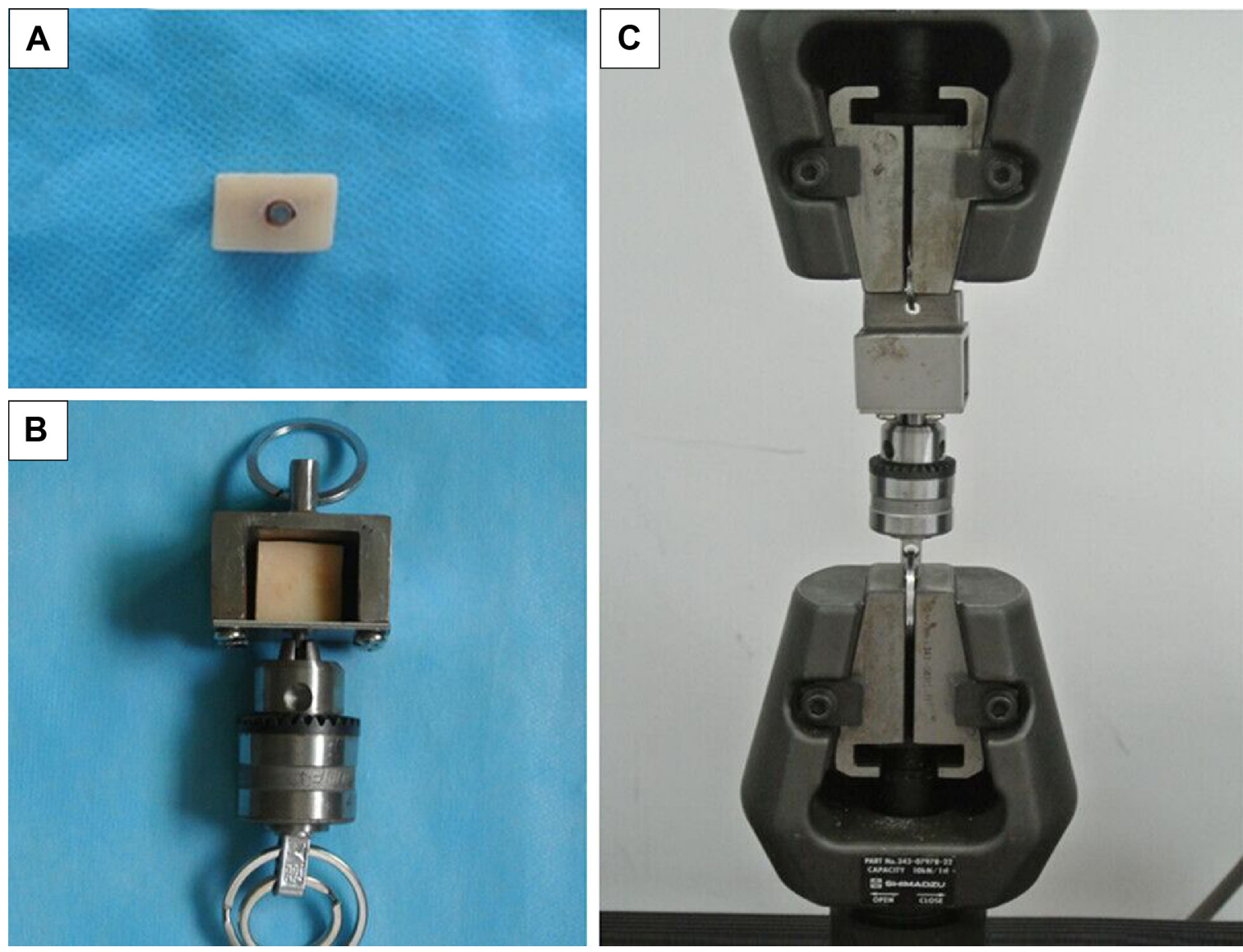

Figure I Procedures of the pull-out test.

Notes: (A) The implant-bone blocks were embedded in poly(methyl methacrylate) resin, (B) the sample was fixed in a tensile test box, and (C) the box was stabilized in the electronic universal material test machine.

of $1.0 \mathrm{~mm} / \mathrm{min}$. Ultimate interfacial strength $(\sigma)$ was calculated using the formula: $\sigma=P /(\pi d h)$, where $P$ is the ultimate pull-out load $(\mathrm{N}), d(\mathrm{~mm})$ is the diameter of the implant, and $h(\mathrm{~mm})$ is the length of the implant in the bone.

After the pull-out test, the implants were fixed in 4\% formaldehyde solution and dehydrated in graded ethanol solutions from $75 \%$ to $100 \%$. The fracture surfaces of boneimplant interface were examined by SEM for the failure mode analyses.

\section{Statistical analysis}

All data are expressed as mean \pm standard deviation, wherever applicable. Statistical analyses were performed using the Student's $t$-test for the purpose of multiple comparisons. Differences were considered significant at $P<0.05$.

\section{Results}

\section{Characterization of BBF-PLLA-NPs}

The BBF-PLLA-NPs were spherical in shape without any aggregation or adhesion (Figure 2A). At high magnification, the BBF-PLLA-NPs showed a smooth surface, on which no pores and cracks were observed (Figure 2B). Moreover, particle size measured by dynamic light scattering revealed that the NPs had a mean particle size of $408 \pm 14 \mathrm{~nm}$ (Figure 2C).

\section{Surface morphology of the antibacterial coating on Ti implant}

The surface morphology of smooth Ti implant, MAOtreated Ti implants, and the antibacterial coating containing BBF-PLLA-NPs is shown in Figure 3. The surface of smooth Ti implant showed a very smooth surface (Figure 3A). After MAO treatment, considerable porous morphology with 1-3 $\mu \mathrm{m}$ diameter appeared on Ti implant (Figure 3B). By cross-linking with gelatin, the BBF-PLLA-NPs were well distributed in the pores of MAO coating (Figure 3C).

\section{Clinical measurements}

Table 1 shows the values of PD, PISF, and MOI of different groups. No significant difference was detected between the experimental group and positive control group for each kind of measurement $(P>0.05)$, but the indexes of blank 

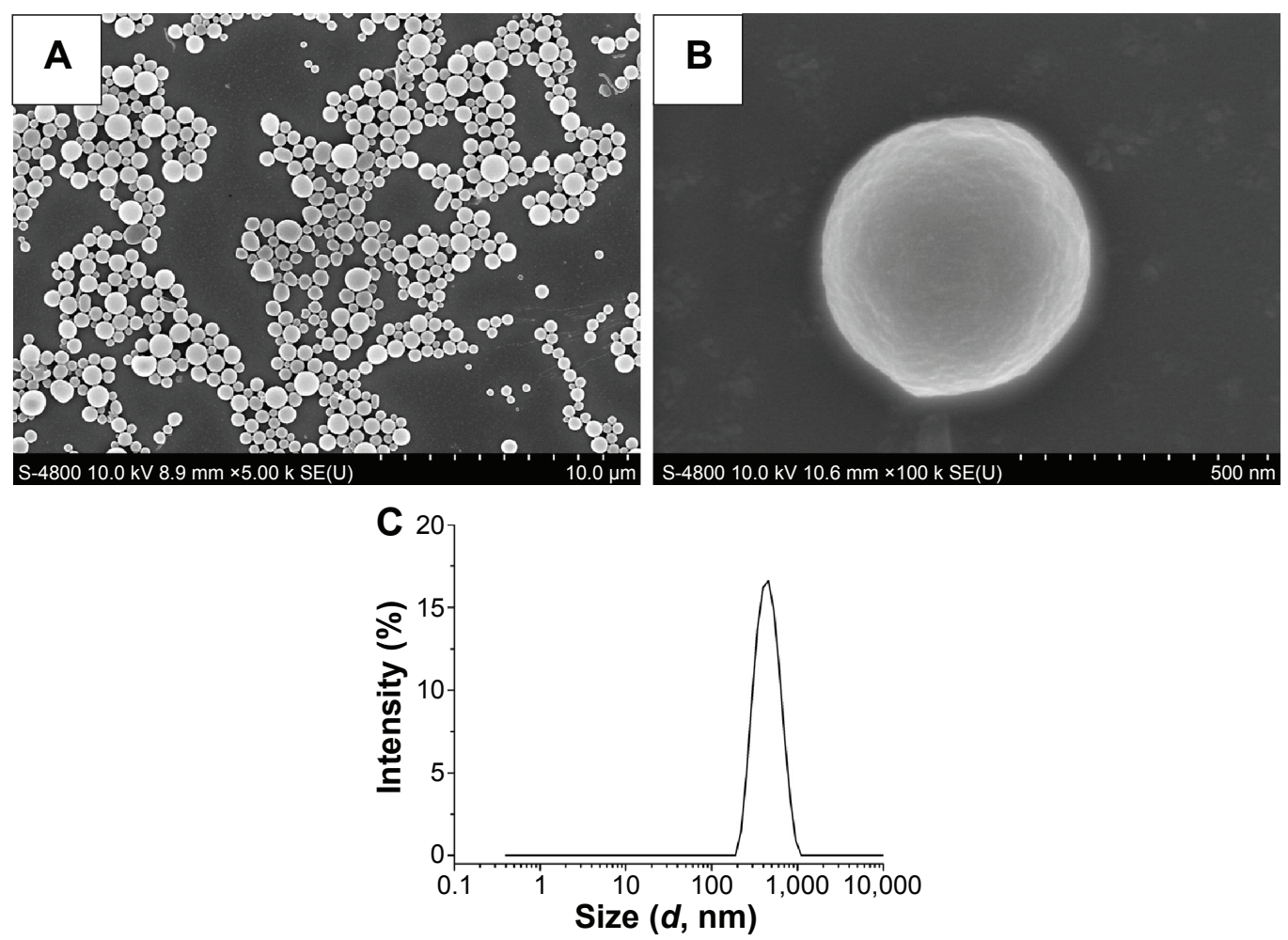

Figure 2 Characterization of BBF-loaded PLLA nanoparticles.

Notes: (A) Scanning electron microscope image at low magnification, (B) scanning electron microscope image at high magnification, and (C) size distribution. Abbreviations: BBF, (Z-)-4-bromo-5-(bromomethylene)-2(5H)-furanone; PLLA, poly(L-lactic acid).
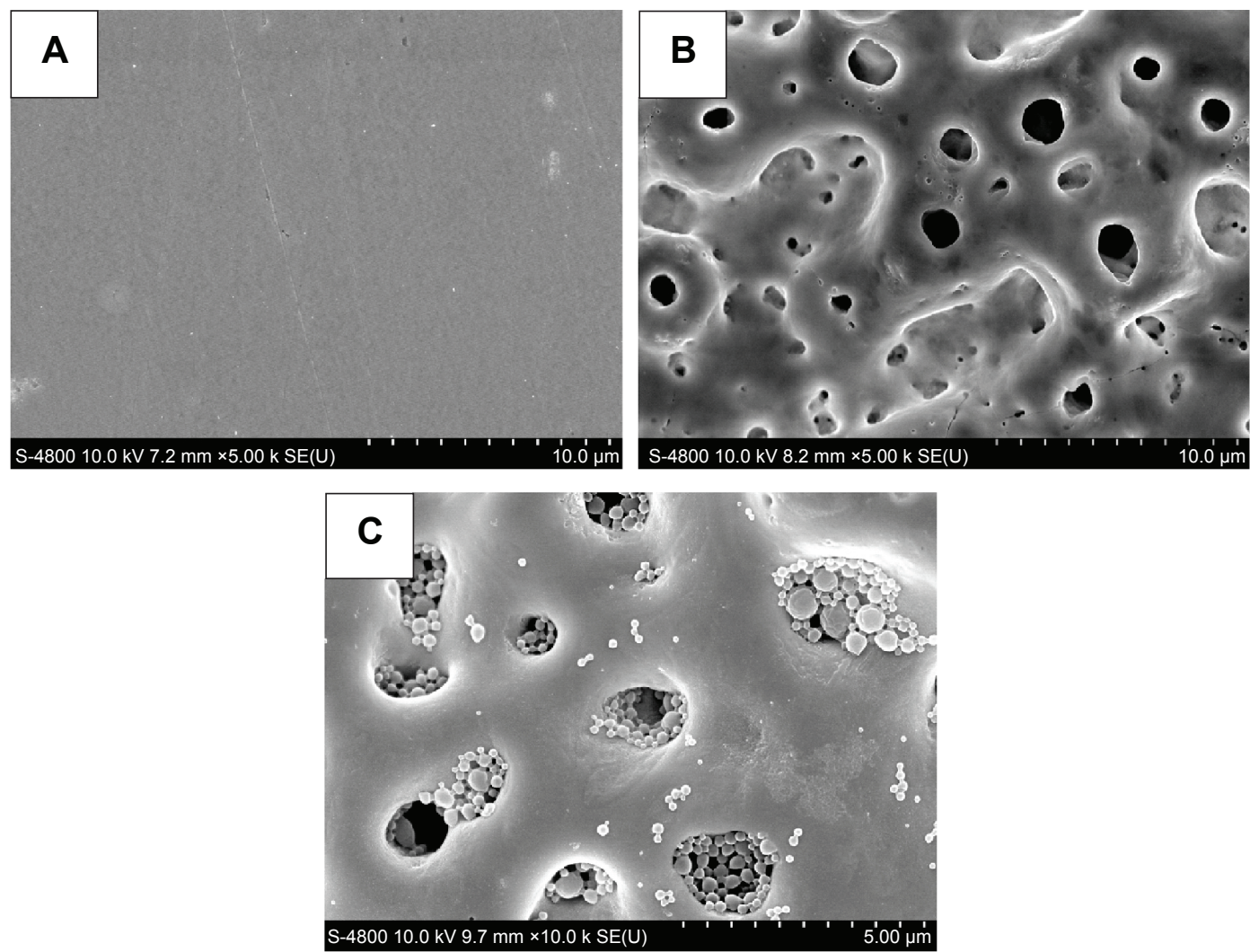

Figure 3 Surface morphology observed by scanning electron microscope.

Notes: (A) Smooth Ti implant, (B) MAO-treated Ti implants, and (C) the antibacterial coating.

Abbreviations: $\mathrm{Ti}$, titanium; MAO, microarc-oxidized. 
Table I Clinical measurements for different groups after 2-month implantation $(x \pm s)$

\begin{tabular}{llll}
\hline Groups & PD $(\mathrm{mm})$ & PISF $(\mu \mathrm{L})$ & MOI \\
\hline Experimental group & $2.65 \pm 0.26$ & $0.96 \pm 0.14$ & $2.22 \pm 0.58$ \\
Positive control group & $2.78 \pm 0.30$ & $0.89 \pm 0.15$ & $2.5 \mathrm{I} \pm 0.64$ \\
Blank control group & $4.50 \pm 0.4 \mathrm{I}^{*}$ & $1.72 \pm 0.25^{*}$ & $5.43 \pm 0.65^{*}$ \\
\hline
\end{tabular}

Note: $* p<0.05$.

Abbreviations: PD, probing depth; PISF, peri-implant sulcular fluid; MOI, mobility of implants.

control group were significantly higher than those of the aforementioned two groups $(P<0.05)$.

Figure 4 shows the $\mathrm{X}$-ray radiographs of the mandible third premolars before extraction (Figure 4A), immediately after implantation immediately (Figure 4B), and after 2-month implantation. It can be clearly seen that there was no obvious shadow around the implants of the experimental and positive control groups (Figure 4C and D), but significant shadow was observed in the blank control group (Figure 4E), and even one implant was loosen and fell off from the mandible (Figure 4F).

\section{Bone-implant interface observation}

Figure 5 shows the scanning electron microscope images of the polished cross-sections of the implants in bone of different groups after 2-month implantation. It showed that the bone apposed directly and tightly to the Ti implants' surface in the experimental and positive control groups (Figure 5A and B). However, a gap was still present between the boneimplant interface in the blank control group (Figure 5C).

From the Van Gieson's staining images (Figure 6), the blank control group demonstrated that more fibrous connective tissues are in contact with the implants' surface (Figure 6C), whereas in the experimental and positive control groups, the bone tissues are in direct contact with the
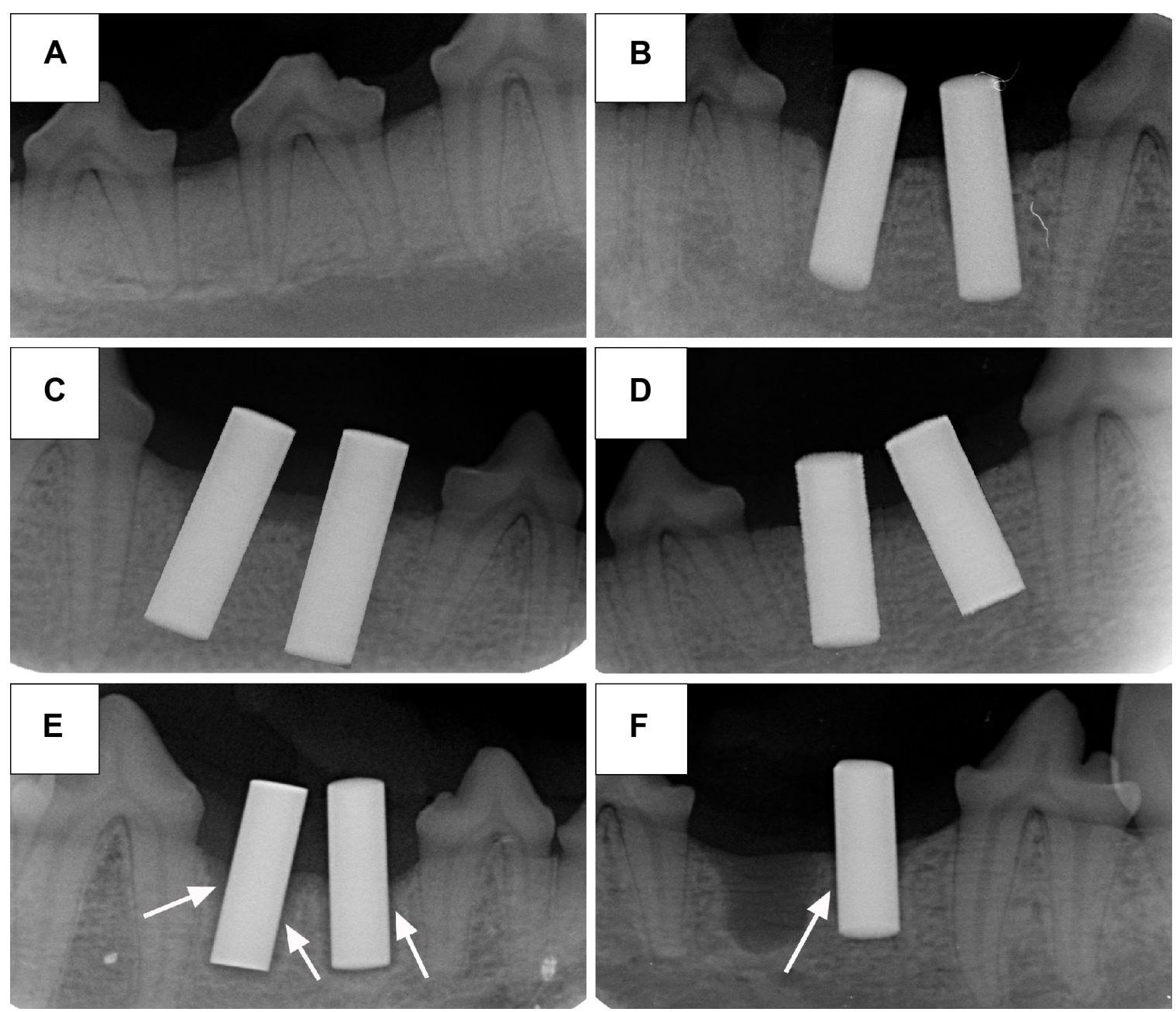

Figure 4 X-ray radiographs.

Notes: (A) The mandible third premolars before extraction, (B) implants after implantation immediately, (C) experimental group after 2-month implantation, (D) positive control group after 2-month implantation, and (E) and (F) blank control group after 2-month implantation. The arrows show the shadow around the implants. 

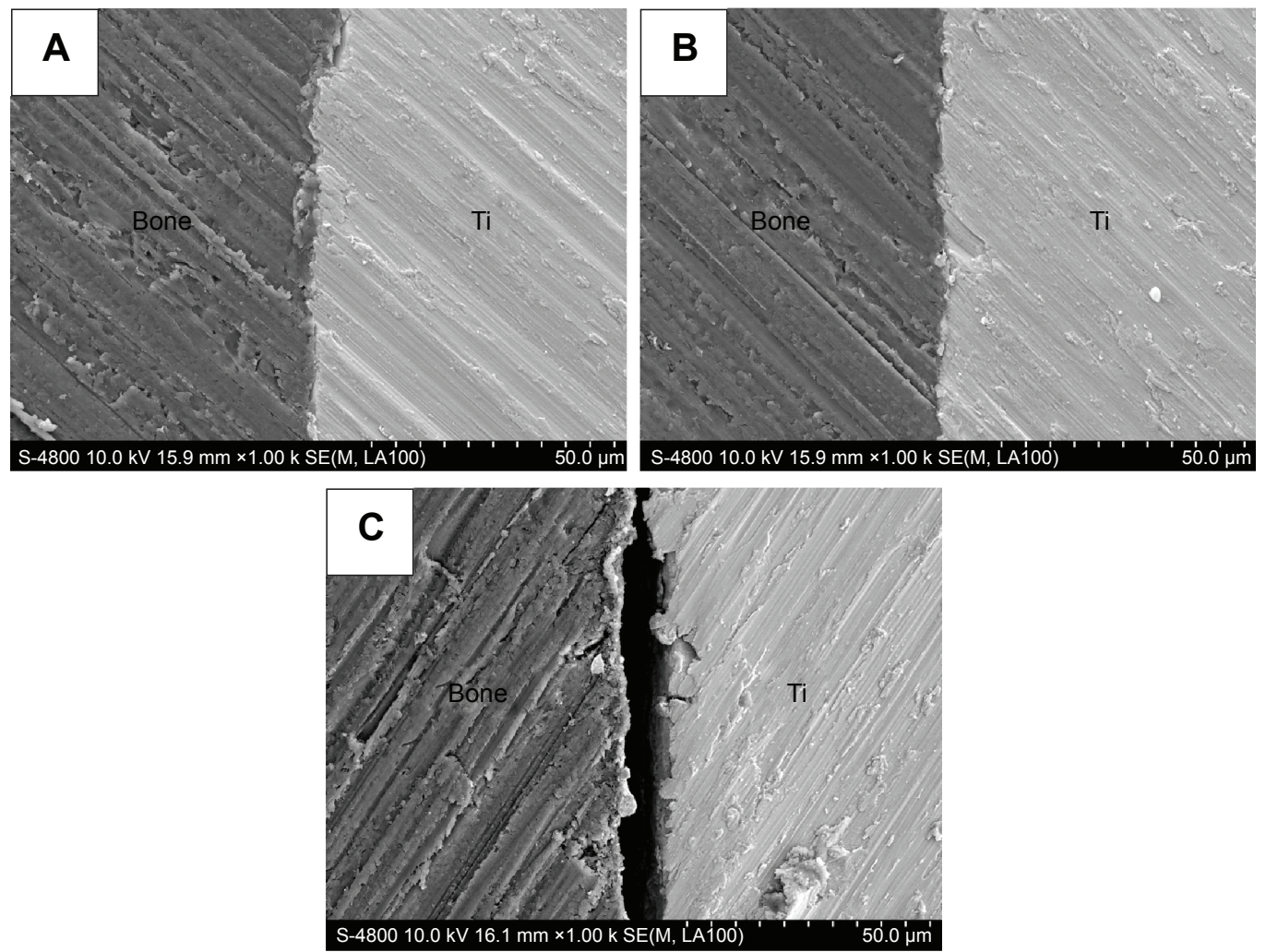

Figure 5 Scanning electron microscope images of polished cross-sections of the implants in bone of different groups after 2-month implantation. Notes: (A) Experimental group, (B) positive control group, and (C) blank control group. Abbreviation: $\mathrm{Ti}$, titanium.
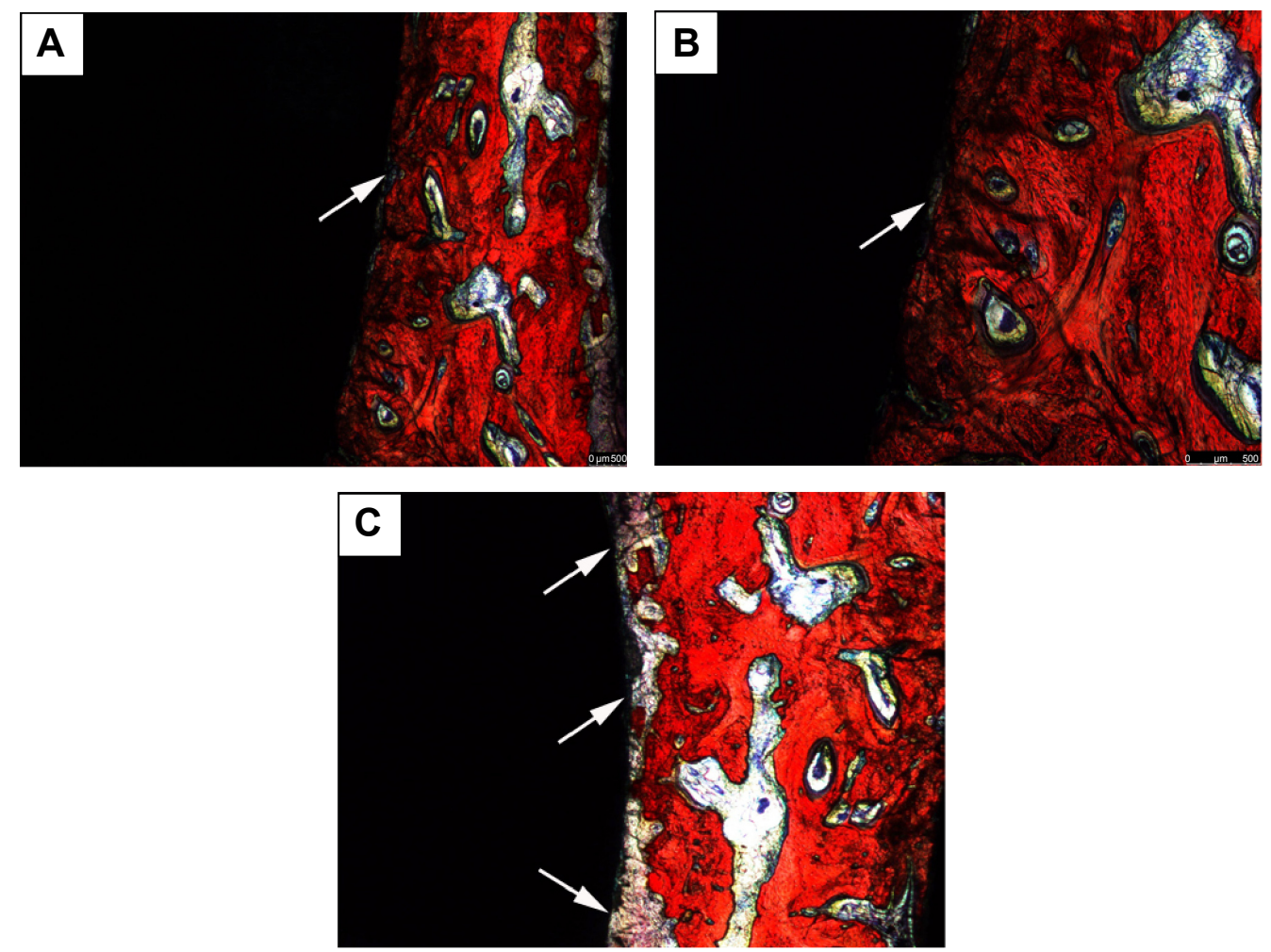

Figure 6 Van Gieson's staining images of polished cross-sections of the implants in bone of different groups after 2-month implantation. Notes: (A) Experimental group, (B) positive control group, and (C) blank control group. The arrows show the fibrous connective tissue. 


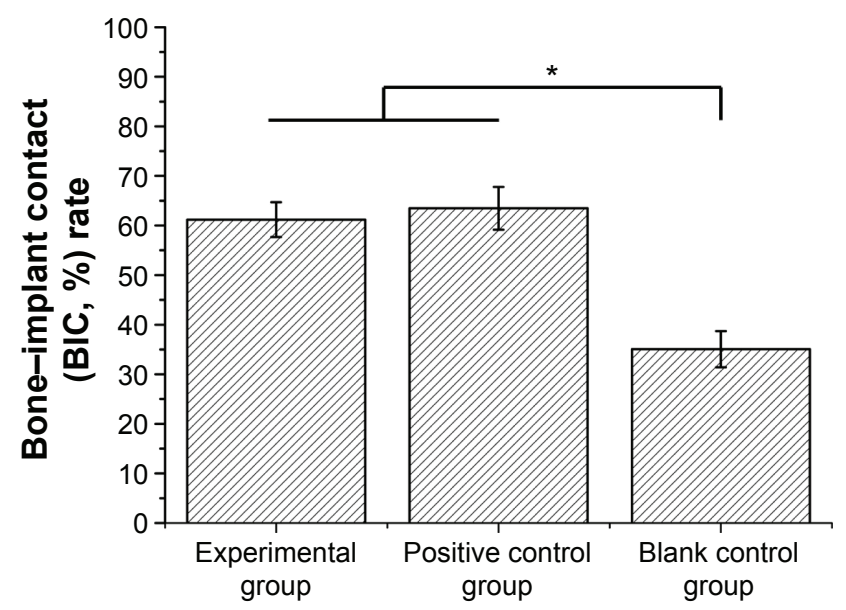

Figure 7 Bone-implant contact (BIC, \%) rate of different groups after 2-month implantation measured by image analysis techniques.

Note: $* P<0.05$.

implants' surfaces (Figure 6A and B). The BIC rates (\%) measured by image analysis techniques are summarized in Figure 7. The BIC values were $61.20 \% \pm 3.51 \%$ and $63.47 \% \pm 4.30 \%$ for implants of the experimental and positive control groups, respectively, after 2 months implantation, which were significantly higher than that of blank control group $(35.06 \% \pm 3.64 \%)(P<0.05)$.

\section{Pull-out test}

The results of the pull-out test are shown in Figure 8. No significant difference in the ultimate interfacial strength after 2 months implantation was observed between the experimental group $(2.40 \pm 0.13 \mathrm{MPa})$ and positive control group $(2.45 \pm 0.21 \mathrm{MPa})(P>0.05)$, which were significantly higher than that of blank control group $(1.03 \pm 0.32 \mathrm{MPa})(P<0.05)$. Figure 9 shows the scanning electron microscope images of the bone-implant interface after the pulled-out test. It was

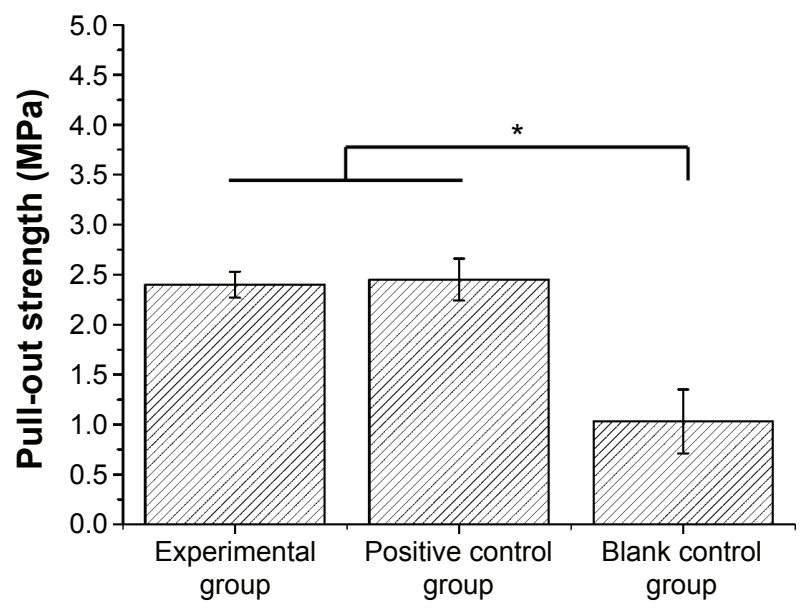

Figure 8 Pull-out strength of different groups after 2-month implantation. Note: $* P<0.05$. observed that some bone remained adhered onto the surface of the implants of the experimental and positive control groups (Figure 9A and B), which indicated that the pull-out strength between the bone-implant interface was higher than the bone itself. There was nearly no bone remained adhered onto the surface of the blank control group (Figure 9C).

\section{Discussion}

Dental implants are the most innovative and superior treatment used in dentistry and are becoming increasingly one of the major treatments for missing teeth. However, periimplant infection arises much attention and still remains a serious challenge in clinic. In recent years, many surface coatings containing or releasing antimicrobial, such as cephalothin, amoxicillin, gentamicin, tobramycin, and vancomycin, have been incorporated into implants with some success. ${ }^{11,24}$ Nevertheless, with the emergence of bacterial drug resistance, ${ }^{25}$ this form of therapy may be short lived and may soon become ineffective. Additionally, a report has pointed out that some antimicrobial agents may cause cell damage. ${ }^{26}$ Chlorhexidine has been widely used in dentistry for the treatment of periodontal infection for its broad spectrum of antimicrobial action and low risk of drug resistance. Studies have absorbed chlorhexidine to the $\mathrm{TiO}_{2}$ layer on the titanium surface, but the gradual release period is only several days. ${ }^{27}$ Silver has also been introduced into titania nanotubes to enhance the bactericidal ability, ${ }^{28}$ nevertheless, its bactericidal mechanism needs further clarification. Thus, novel implant antibacterial-coating system is important. Halogenated furanones, a new kind of antibacterial agent, posses stable antimicrobial activity against a great number of bacteria, ${ }^{19}$ while less prone to resistance development. ${ }^{20}$ Because BBF was one of the most active halogenated furanone compounds, ${ }^{29}$ it was used in this study. To achieve the goal of sustained release, BBF was incorporated into PLLA-NPs, and then we developed a method to fabricate a new antibacterial coating, by cross-linking the BBF/ PLLA-NPs on MAO Ti. ${ }^{22}$ Observed under SEM, the BBFPLLA-NPs were well distributed and cross-linked with each other and wall of pores by physical interlocking with gelatin (Figure 3C). This topographic character indicated that the antibacterial coating had relatively high stability and binding capacity, which may effectively prevent the antibacterial NPs from falling off during the dental implant surgery. The antibacterial experiment in vitro showed that the antibacterial coating exhibited relatively long-term antibacterial ability. ${ }^{23}$ Thus, the purpose of this study is to identify whether the antibacterial containing BBF-PLLA-NPs has 

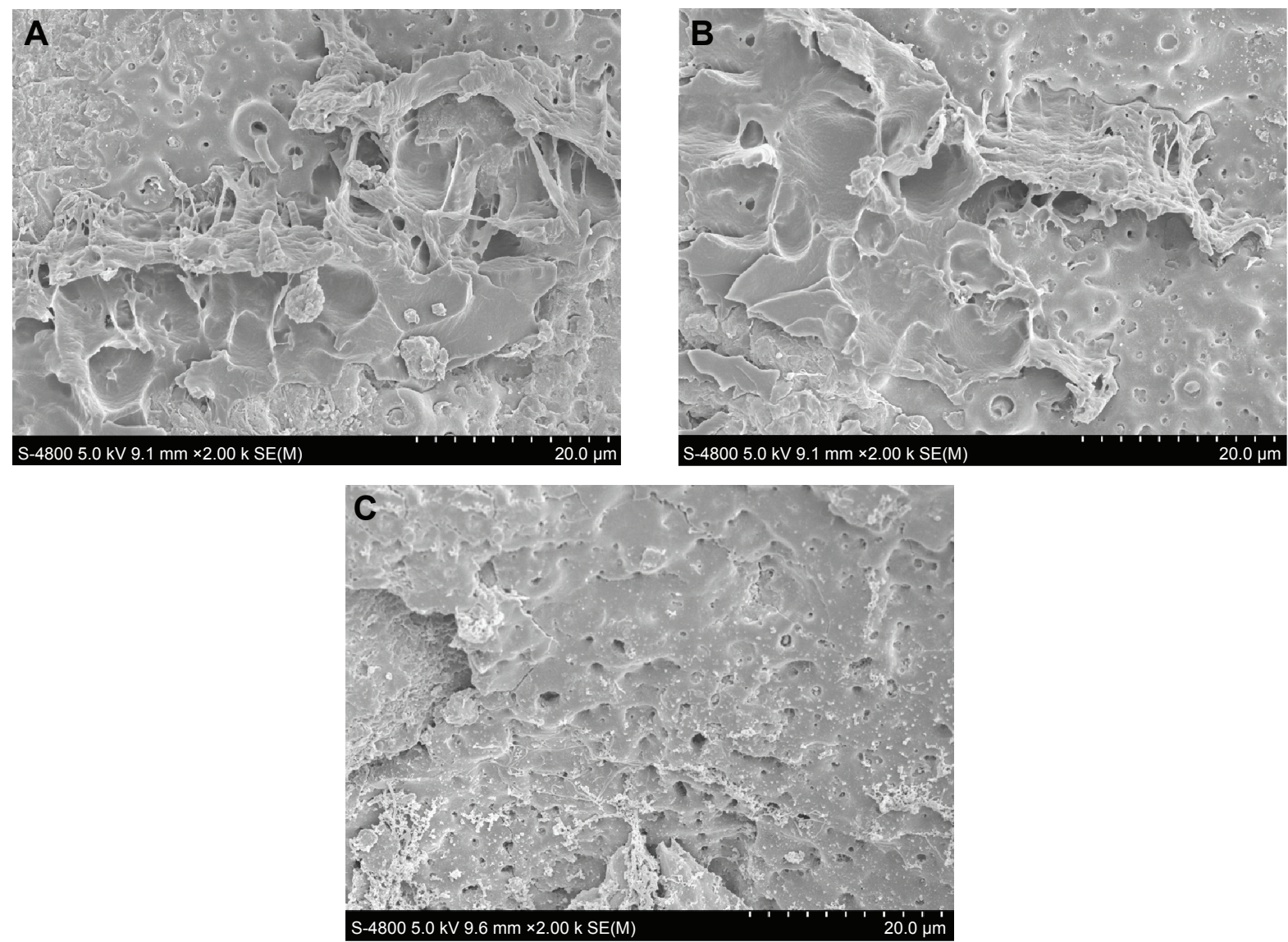

Figure 9 Scanning electron microscope images of the bone-implant interface of different groups after the pulled-out test. Notes: (A) Experimental group, (B) positive control group, and (C) blank control group.

positive effect on the prevention of peri-implant infection under a simulated environment of peri-implant infection in vivo and to justify its further evaluation for clinical applications.

In this study, after tooth extraction, the implants were immediately placed. Immediate implant placement often reduces the treatment time and expense for patients, and a comparative clinical study has found that implant survival rates after immediate placement are similar to those after delayed placement. ${ }^{30}$ After implantation, the peri-implant infection was simulated by ligaturing $4-0$ wire around the implants upon the gums and feeding high sugar diet $(100 \mathrm{~g} /$ $\mathrm{kg}$ ). You et $\mathrm{al}^{31}$ also used this method to induce peri-implant infection. Minocycline hydrochloride ointment is a commonly used sustained-release topical antibiotic for periodontitis. ${ }^{32}$ Since the microbial flora of peri-implant infection is traditionally associated with periodontitis, ${ }^{5}$ minocycline hydrochloride ointment is often used for peri-implant infection. ${ }^{33}$ Therefore, after implantation, minocycline hydrochloride ointment was used once a week according to the directions for the positive control group in this study.
Early osseointegration is crucial to the success of implant, so it is very important to prevent peri-implant in the early stage after surgery. In the current study, MAO Ti implants loaded with BBF-PLLA-NPs showed excellent preventive effect under the simulated environment of peri-implant infection in the early stage. For clinical measurements, the PD, PISF, and MOI were examined. Lang et $\mathrm{al}^{34}$ demonstrated that the clinical measurements reveal the correlation to the level of attachment of the implant. It may also provide a good indicator to evaluate the status of health or disease of peri-implant tissues. In healthy condition, the PD of periimplant pocket is $<3 \mathrm{~mm}$. When the peri-implant infection occurred, the PD would increase. ${ }^{35}$ In this study, the PD of the experimental and positive control groups was $<3 \mathrm{~mm}$, while the PD of blank control group was $>3 \mathrm{~mm}$. The PISF was a sensitive parameter of peri-implant infection. The PISF would also increase after peri-implant infection. ${ }^{36}$ The PISF of blank control group was $1.72 \pm 0.25 \mu \mathrm{L}$ in this study, which was much higher than those of the experimental and positive control groups. Periotest was a simple method to measure the MOI. The value of MOI of the blank control group was 
much higher than that of the other two groups, which indicated that the implants were more loose. These results of clinical measurements proved that the experimental group had remarkable preventive effect on peri-implant infection as the positive control group.

Peri-implant infection would lead to the absorption of bone tissue around the implants, which is observed as a shadow on the X-ray radiograph. Therefore, significant shadow was observed on the X-ray radiograph of the blank control group (Figure 4E), and one implant even loosened and fell off from the mandible (Figure 4F).

Scanning electron microscope images and Van Gieson's staining images of the polished cross-sections of the implants in bone of different groups demonstrated that the implants with antibacterial coating and positive control group exhibited a tighter osseointegration than the blank control group (Figures 5 and 6), which was benefited by the antibacterial effect. The BIC measured by image analysis techniques also indicated that more BIC occurred in the experimental and positive control groups (Figure 7).

Along with the histological examination, the actual effect of preventing infection of the antibacterial coating was further investigated by pull-out test. The ultimate interfacial strength of the experimental group and positive control group was significantly higher than that of the blank control group (Figure 8). Theoretically, there are seven kinds of interfacial bond failure exist between bone and implant when mechanical loads applied including 1) at the titanium substrate/coated materials (oxide coating), 2) inside the coating materials, 3 ) at the interface implant surface (oxide)/immature bone, 4) inside the immature bone, 5) between the immature bone and the surrounding mature bone, 6) in the surrounding bone, and 7) any combined fracture types - heterogeneous fracture mode. ${ }^{37}$ The actual bond failure is determined by the biochemical bonding strength. In this pull-out test, the SEM revealed that the fracture surface of the blank control group should be between the bone-implant interface (Figure 9C). For the experimental and positive control groups, the remaining bone fragments showed that the fracture mainly occurred in the bone (Figure 9A and B), which indicated that the biochemical bonding strength of the implants in the experimental and positive control groups was stronger than the bonding strength between the bone tissues. This phenomenon also proved that the novel antibacterial coating containing BBF-PLLA-NPs possessed stable preventive effect under the simulated environment of peri-implant infection after 2-month implantation.
It was amazing to observe that the experimental and positive control groups showed almost the same preventive effect. Minocycline hydrochloride ointment is often used for peri-implant infection. ${ }^{33}$ Of note, minocycline hydrochloride ointment was used once a week after implantation. But, the implants coated with this antibacterial coating did not need to do anything after implantation, which was benefited by the long-term antibacterial ability. Thus, if this method could be applied clinically to prevent peri-implant infection, it would greatly reduce the patients' clinic visits.

\section{Conclusion}

The antibacterial coating showed remarkable preventive effect under the simulated environment of peri-implant infection in clinical measurements, bone-implant interface, percentage of bone contact, and implant binding strength in vivo. It can be concluded that the fabricated antibacterial coating on $\mathrm{Ti}$ implants may be a potential and promising strategy to prevent peri-implant infection at the early stage after implantation.

\section{Acknowledgments}

This work was supported by the National Natural Science Foundation of China (grant number 51371006) and Medical Science and Technology Innovation Fund Project of Nanjing Military Region (grant number 15MS058).

\section{Disclosure}

The authors report no conflicts of interest in this work.

\section{References}

1. Ferrigno N, Laureti M, Fanali S, Grippaudo G. A long-term follow-up study of non-submerged ITI implants in the treatment of totally edentulous jaws. Part I: ten-year life table analysis of a prospective multicenter study with 1286 implants. Clin Oral Implants Res. 2002;13(3): 260-273.

2. Simonis $P$, Dufour T, Tenenbaum H. Long-term implant survival and success: a 10-16-year follow-up of non-submerged dental implants. Clin Oral Implants Res. 2010;21(7):772-777.

3. Palma-Carrio C, Maestre-Ferrin L, Penarrocha-Oltra D, PenarrochaDiago MA, Penarrocha-Diago M. Risk factors associated with early failure of dental implants. A literature review. Med Oral Patol Oral Cir Bucal. 2011;16(4):e514-e517.

4. Schwartz-Arad D, Laviv A, Levin L. Failure causes, timing, and cluster behavior: an 8-year study of dental implants. Implant Dent. 2008;17(2): 200-207.

5. Pye AD, Lockhart DE, Dawson MP, Murray CA, Smith AJ. A review of dental implants and infection. $J$ Hosp Infect. 2009;72(2):104-110.

6. Paster BJ, Boches SK, Galvin JL, et al. Bacterial diversity in human subgingival plaque. J Bacteriol. 2001;183(12):3770-3783.

7. Quirynen M, Teughels W. Microbiologically compromised patients and impact on oral implants. Periodontol 2000. 2003;33:119-128.

8. Hu H, Zhang W, Qiao Y, Jiang X, Liu X, Ding C. Antibacterial activity and increased bone marrow stem cell functions of $\mathrm{Zn}$-incorporated $\mathrm{TiO} 2$ coatings on titanium. Acta Biomater. 2012;8(2):904-915. 
9. Donlan RM, Costerton JW. Biofilms: survival mechanisms of clinically relevant microorganisms. Clin Microbiol Rev. 2002;15(2):167-193.

10. Zhao L, Chu PK, Zhang Y, Wu Z. Antibacterial coatings on titanium implants. J Biomed Mater Res B Appl Biomater. 2009;91(1): 470-480.

11. Stigter M, Bezemer J, de Groot K, Layrolle P. Incorporation of different antibiotics into carbonated hydroxyapatite coatings on titanium implants, release and antibiotic efficacy. J Control Release. 2004; 99(1):127-137.

12. Chua PH, Neoh KG, Kang ET, Wang W. Surface functionalization of titanium with hyaluronic acid/chitosan polyelectrolyte multilayers and RGD for promoting osteoblast functions and inhibiting bacterial adhesion. Biomaterials. 2008;29(10):1412-1421.

13. Zhao L, Wang H, Huo K, et al. Antibacterial nano-structured titania coating incorporated with silver nanoparticles. Biomaterials. 2011;32(24): 5706-5716.

14. Feres M, Haffajee AD, Allard K, Som S, Goodson JM, Socransky SS. Antibiotic resistance of subgingival species during and after antibiotic therapy. J Clin Periodontol. 2002;29(8):724-735.

15. Antoci VJ, Adams CS, Hickok NJ, Shapiro IM, Parvizi J. Antibiotics for local delivery systems cause skeletal cell toxicity in vitro. Clin Orthop Relat Res. 2007;462:200-206.

16. Naal FD, Salzmann GM, von Knoch F, et al. The effects of clindamycin on human osteoblasts in vitro. Arch Orthop Trauma Surg. 2008;128(3) 317-323.

17. de Nys R, Givskov M, Kumar N, Kjelleberg S, Steinberg PD. Furanones. Prog Mol Subcell Biol. 2006;42:55-86.

18. Ren D, Bedzyk LA, Ye RW, Thomas SM, Wood TK. Differential gene expression shows natural brominated furanones interfere with the autoinducer-2 bacterial signaling system of Escherichia coli. Biotechnol Bioeng. 2004;88(5):630-642.

19. Manefield M, Rasmussen TB, Henzter M, et al. Halogenated furanones inhibit quorum sensing through accelerated LuxR turnover. Microbiology. 2002;148(pt 4):1119-1127.

20. Raffa RB, Iannuzzo JR, Levine DR, et al. Bacterial communication ("quorum sensing") via ligands and receptors: a novel pharmacologic target for the design of antibiotic drugs. J Pharmacol Exp Ther. 2005; 312(2):417-423

21. Baveja JK, Li G, Nordon RE, et al. Biological performance of a novel synthetic furanone-based antimicrobial. Biomaterials. 2004;25(20): 5013-5021.

22. Cheng Y, Wu J, Gao B, et al. Fabrication and in vitro release behavior of a novel antibacterial coating containing halogenated furanoneloaded poly(L-lactic acid) nanoparticles on microarc-oxidized titanium. Int J Nanomedicine. 2012;7:5641-5652.

23. Cheng Y, Zhao X, Liu X, et al. Antibacterial activity and biological performance of a novel antibacterial coating containing a halogenated furanone compound loaded poly(L-lactic acid) nanoparticles on microarc-oxidized titanium. Int J Nanomedicine. 2015;10:727-737.
24. Gautier H, Daculsi G, Merle C. Association of vancomycin and calcium phosphate by dynamic compaction: in vitro characterization and microbiological activity. Biomaterials. 2001;22(18):2481-2487.

25. Stewart PS, Costerton JW. Antibiotic resistance of bacteria in biofilms Lancet. 2001;358(9276):135-138.

26. Harris LG, Mead L, Muller-Oberlander E, Richards RG. Bacteria and cell cytocompatibility studies on coated medical grade titanium surfaces. J Biomed Mater Res A. 2006;78(1):50-58.

27. Kozlovsky A, Artzi Z, Moses O, Kamin-Belsky N, Greenstein RB Interaction of chlorhexidine with smooth and rough types of titanium surfaces. J Periodontol. 2006;77(7):1194-1200.

28. Mei S, Wang H, Wang W, et al. Antibacterial effects and biocompatibility of titanium surfaces with graded silver incorporation in titania nanotubes. Biomaterials. 2014;35(14):4255-4265.

29. Janssens JC, Steenackers H, Robijns S, et al. Brominated furanones inhibit biofilm formation by Salmonella enterica serovar typhimurium. Appl Environ Microbiol. 2008;74(21):6639-6648.

30. Al-Sabbagh M, Kutkut A. Immediate implant placement: surgical techniques for prevention and management of complications. Dent Clin North Am. 2015;59(1):73-95.

31. You TM, Choi BH, Zhu SJ, et al. Treatment of experimental periimplantitis using autogenous bone grafts and platelet-enriched fibrin glue in dogs. Oral Surg Oral Med Oral Pathol Oral Radiol Endod. 2007;103(1):34-37.

32. Javed S, Kohli K. Local delivery of minocycline hydrochloride: a therapeutic paradigm in periodontal diseases. Curr Drug Deliv. 2010;7(5): 398-406.

33. Mombelli A, Feloutzis A, Bragger U, Lang NP. Treatment of peri-implantitis by local delivery of tetracycline. Clinical, microbiological and radiological results. Clin Oral Implants Res. 2001;12(4): 287-294.

34. Lang NP, Wetzel AC, Stich H, Caffesse RG. Histologic probe penetration in healthy and inflamed peri-implant tissues. Clin Oral Implants Res. 1994;5(4):191-201.

35. Abrahamsson I, Soldini C. Probe penetration in periodontal and periimplant tissues. An experimental study in the beagle dog. Clin Oral Implants Res. 2006;17(6):601-605.

36. Niimi A, Ueda M. Crevicular fluid in the osseointegrated implant sulcus: a pilot study. Int J Oral Maxillofac Implants. 1995;10(4):434-436.

37. Steketee M, Balazovich K, Tosney KW. Filopodial initiation and a novel filament-organizing center, the focal ring. Mol Biol Cell. 2001; 12(8):2378-2395.
International Journal of Nanomedicine

\section{Publish your work in this journal}

The International Journal of Nanomedicine is an international, peerreviewed journal focusing on the application of nanotechnology in diagnostics, therapeutics, and drug delivery systems throughou the biomedical field. This journal is indexed on PubMed Central, MedLine, CAS, SciSearch $\AA$, Current Contents ${ }^{\circledR} /$ Clinical Medicine,

\section{Dovepress}

Journal Citation Reports/Science Edition, EMBase, Scopus and the Elsevier Bibliographic databases. The manuscript management system is completely online and includes a very quick and fair peer-review system, which is all easy to use. Visit http://www.dovepress.com/ testimonials.php to read real quotes from published authors. 